BULLETIN (New Series) OF THE

AMERICAN MATHEMATICAL SOCIETY

Volume 46, Number 2, April 2009, Pages 309-326

S 0273-0979(09)01244-0

Article electronically published on January 28, 2009

\title{
ON THE ORIGIN AND DEVELOPMENT OF SUBFACTORS AND QUANTUM TOPOLOGY
}

\author{
VAUGHAN JONES
}

AbStRact. We give an account of the beginning of subfactor theory and TQFT and some more recent developments.

\section{INTRODUCTION}

In 2008 the Mathematical Sciences Research Institute at Berkeley celebrated the 25th anniversary of its opening. Among the celebrations there were talks dealing with some of the programs MSRI has run over the years. This article is a slightly enhanced version of the talk I gave which recalled the 1984-1985 programs on operator algebras and low dimensional topology and briefly surveyed some of the outcomes of the interaction between these two programs. These include the subject of topological quantum field theory (TQFT) and the interactions of subfactor theory with physics, but I do not wish to suggest that these areas have been more important than other developments in operator algebras or topology - it is just that they have been the ones I have been most involved in.

In writing this article it was immediately obvious that any kind of comprehensive survey of either operator algebraic developments or topological ones would be a huge task, so I have tried to preserve the informal nature of the talk, no doubt preserving some of its inaccuracies and incompleteness, but hopefully never being misleading. The article is entirely unbalanced in the way it treats the early developments and the more recent ones, but I have included many references that should allow the reader to track down what has been going on. I apologise to the many people who have made major contributions that are not mentioned here.

\section{BEFORE THE 1984-1985 MSRI YEAR}

Not long before the MSRI year there had been the rediscovery in subfactors of the "Temperley-Lieb" algebra, as presented by the following generators and relations:

$$
\begin{aligned}
e_{i}^{2}=e_{i}^{*}=e_{i}, & i=1,2, \cdots, n, \\
e_{i} e_{j}=e_{j} e_{i}, & \text { if }|i-j| \geq 2, \\
e_{i} e_{i \pm 1} e_{i}=\tau e_{i}, & \text { for } \quad 1 \leq i<n .
\end{aligned}
$$

Received by the editors November 12, 2008.

2000 Mathematics Subject Classification. Primary 46L37; Secondary 46L54.

Key words and phrases. Subfactors, planar algebras, TQFT, knots, CFT, statistical mechanics. Supported by NSF under Grant No. DMS-0401734, Auckland University and the NZIMA.

(C)2009 American Mathematical Society Reverts to public domain 28 years from publication 
The first relation means that when represented on Hilbert space the $e_{i}$ are $n$ orthogonal projections onto closed subspaces. The second says that the subspaces are orthogonal modulo their intersection if $|i-j| \geq 2$. The third says that the "angle" between $i$ th and $(i+1)$ th subspaces is determined by the number $\tau \in \mathbb{R}$. Thus these three relations are purely geometric in nature. The subfactor context also provided a trace tr on the algebra generated by the $e_{i}$ 's uniquely defined by

$$
\operatorname{tr}\left(w e_{n+1}\right)=\tau \operatorname{tr}(w) \quad \text { if } w \text { is a word on } e_{1}, e_{2}, \ldots, e_{n} .
$$

By a geometric or an algebraic analysis it can be shown that for infinitely many $e_{i}$ 's to exist as above, $\frac{1}{\tau}$ must be either $\geq 4$ or one of the numbers

$$
4 \cos ^{2} \pi / k \quad \text { for } k=3,4,5, \cdots .
$$

Wenzl in [We87] showed that these restrictions apply even without the trace.

Temperley and Lieb came across almost exactly the same relations (though without the trace) in work on solvable models in two-dimensional statistical mechanics. The $e_{i}$ 's occur as elementary "transfer matrices" corresponding to adding a single interaction between spins on a lattice. They showed that the same algebra underlies the Potts model and Lieb's ice-type model and deduced a mathematical equivalence between the two models. See [TL70] and chapter 12 of [Ba82]. In subfactors the $e_{i}$ 's arose as orthogonal projections in a canonical tower of algebras arising from a subfactor $N \subseteq M$ of a $\mathrm{II}_{1}$ factor $M$. The number $\tau$ in the relations is the reciprocal of what is known as the index $[M: N]$ of $N$ in $M$. The tower of $\mathrm{II}_{1}$ factors is constructed in an inductive way in which each step depends on the last two but is independent of the previous steps. The $e_{i}$ 's are the conditional expectations from each step to the preceding one. See Jon83.

(A $\mathrm{II}_{1}$ factor $M$ is a unital *-algebra of bounded operators on a Hilbert space closed in the topology of pointwise convergence, whose centre is just multiples of the identity and which possesses a trace, a linear function from $M$ to $\mathbb{C}$ which vanishes on commutators. One also requires $M$ to be infinite dimensional since the $n \times n$ matrices satisfy all these conditions. An example is the closure for pointwise convergence of the group algebra of the free group on two or more generators, acting on the Hilbert space of square summable functions on the group in the obvious way.)

The above result immediately shows that the index $[M: N]$ must be $\geq 4$ or in the above set of numbers.

One may also construct by hand infinite families of projections, with a trace, satisfying the above relations. The algebra they generate is a $\mathrm{II}_{1}$ factor. A subfactor realising the appropriate index value is obtained by taking the algebra generated by all the $e_{i}$ 's with $i \geq 2$.

The (finite-dimensinoal) algebras $A_{n}$ defined by $1, e_{1}, e_{2}, \ldots, e_{n}$, are easily described inductively in terms of "Bratteli diagrams" which give the irreducible representations of $A_{n}$ as vertices of a graph with edges according to how a representation of $A_{n}$ restricts to $A_{n-1}$. The main picture is the following truncated Pascal's triangle which works for all $\tau<1 / 4$. 


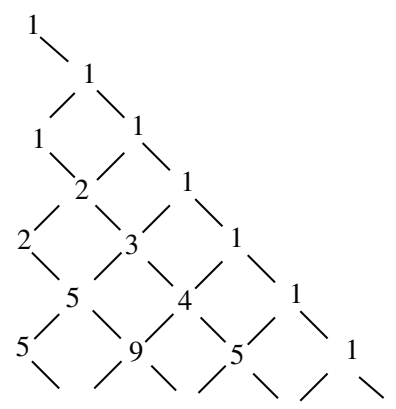

For instance, the 2 and 1 in the third row correspond to a two-dimensional and a one-dimensional irreducible representation of $A_{2}$, the two-dimensional one containing both the one-dimensional irreducible representations of $A_{1}$.

For $1 / \tau=4 \cos ^{2} \pi / k$, one further truncates the diagram on the right by removing a " 1 " and all its descendants. Thus, for instance, for $1 / \tau=4 \cos ^{2} \pi / 7$ one obtains the following.

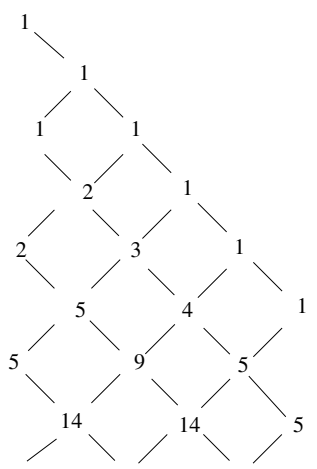

The first irrational index value, $4 \cos ^{2} \pi / 5$, gives a Bratteli diagram with just two columns, all the entries being Fibonacci numbers.

The braid group is the group presented on generators $\sigma_{1}, \sigma_{2}, \cdots, \sigma_{n-1}$, with relations

$$
\begin{gathered}
\sigma_{i} \sigma_{j}=\sigma_{j} \sigma_{i}, \quad \text { if }|i-j| \geq 2, \\
\sigma_{i} \sigma_{i+1} \sigma_{i}=\sigma_{i+1} \sigma_{i} \sigma_{i+1}, \quad \text { for } 1 \leq i<n .
\end{gathered}
$$

Compare these relations with the $e_{i}$ ones above:

$$
\begin{gathered}
e_{i} e_{j}=e_{j} e_{i}, \quad \text { if }|i-j| \geq 2, \\
e_{i} e_{i \pm 1} e_{i}=\tau e_{i}, \quad \text { for } 1 \leq i<n .
\end{gathered}
$$

To get a representation of the braid group, try sending $\sigma_{i}$ to

$$
t e_{i}-\left(1-e_{i}\right) \text {. }
$$

A simple calculation shows that this works iff $\tau=\frac{t}{(1+t)^{2}}$. 
Note that if $e_{i}$ were diagonalised, $\sigma_{i}$ would look look like

$$
\left(\begin{array}{rrrrrr}
t & 0 & 0 & & & \\
0 & t & 0 & \cdots & & \\
0 & 0 & t & & & \\
\cdots & & & & -1 & 0 \\
& & & & 0 & -1
\end{array}\right)
$$

(Note: $0<\tau \leq 1 / 4$ means $0<t<\infty$, while $1 / \tau=4 \cos ^{2} \pi / k$ means $t=e^{ \pm \frac{2 \pi i}{k}}$.)

So these braid group representations are manifestly unitary if $t=e^{ \pm \frac{2 \pi i}{k}}$ and not otherwise. Their decomposition into irreducibles and restrictions from $B_{n}$ to $B_{n-1}$ are of course given by the Bratteli diagrams.

The braid group $B_{n}$ is also given topologically by isotopy classes of ways of joining points on a given horizontal level to the same points on another level. Thus a picture of a braid in $B_{4}$ is as below.

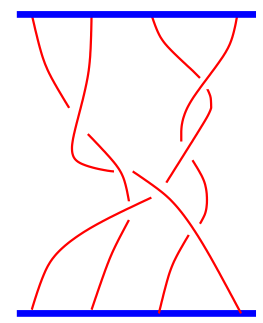

The curves joining the points are called the strings of the braid and they are never allowed to have a horizontal tangent vector. The generators $\sigma_{i}$ above are just the braids with all strings vertical except those joining the $i$ th and $(i+1)$ th points which they join with a single crossing in between, with some convention about positivity of crossings. One may take a braid and turn it into a knot (or link in general) by tying the tops to the bottoms as depicted below.

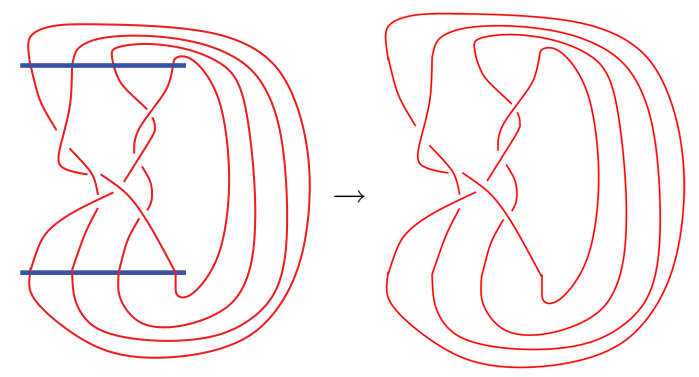

For $\alpha \in B_{n}$, we call $\bar{\alpha}$ the oriented link so obtained. By a theorem of Alexander any oriented link in $\mathbb{R}^{3}$ may be represented in this way.

If $\alpha$ is represented in the Temperley-Lieb algebra as above, by a theorem of Markov (see [Bi75]),

$$
\text { (normalisation) } \operatorname{tr}(\alpha)
$$

gives an invariant of the knot (or link) $\bar{\alpha}$. If the link has an odd number of components it is a Laurent polynomial in $t$, written $V_{L}(t)$ for a link $L$. If the 
number of components is even, $V_{L}(t)$ is $\sqrt{t}$ times a Laurent polynomial in $t$. For the trefoil knot $K$ shown below,

$$
V_{K}(t)=t+t^{3}-t^{4}
$$

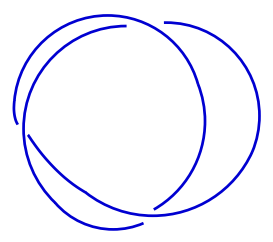

Passing from a knot to its mirror image changes the variable in the polynomial from $t$ to $1 / t$ so this simple polynomial distinguishes between the trefoil and its mirror image.

It is amusing that the key ingredient making the above braid representation-knot invariant work is the compatibility of our trace on the Temperley-Lieb algebra and the changes of braid in Markov's theorem. But the formula defining the trace is also strongly reminiscent of the Markov condition in the theory of Markov chains... different Markov's - both Andrey Andreyevitch - father and son! (Thanks to Garry Tee, University of Auckland, for this information.) The case for naming such a trace a Markov trace is doubly compelling!

This summarises what was known going into the year 1984-1985. Then things moved fast, with most of the action involving MSRI.

\section{MSRI YEAR 1984-1985}

1) The polynomial $V_{L}(t)$ was extended to a two-variable generalisation, the HOMFLYPT polynomial, which also contained the Alexander polynomial (see HOMFLY85).

2) Several applications including braid index estimates and answers to some questions about Conway's "skein theory" (see [FW87, Mor86]).

3) Observation from statistical mechanical models that $V_{L}(t)$ is essentially independent of the orientation of $L$ (see [LM86]). These were soon followed by Kauffman's great diagrammatic insights:

a) $V_{L}(t)$ is essentially an unoriented invariant, simply the result of replacing a crossing by no crossing in both ways:

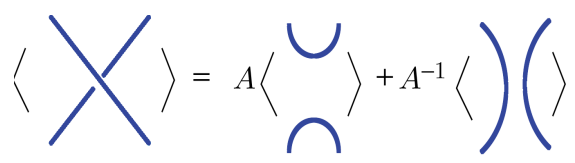

Here the pointed brackets represent the invariant of what is enclosed. It is known as the "Kauffman bracket". Once an orientation is given to the knot, its Kauffman bracket can be easily renormalised to give $V_{L}(t)$ with $t=A^{4}$.

b) The Temperley-Lieb algebra can be realised entirely diagrammatically as pictured below. 


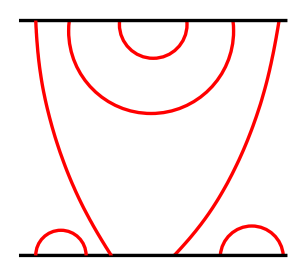

They use braid-like concatenation as multiplication.

Closed loops in the diagrams appear under concatenation and cannot be avoided. But they are simply handled by removing a closed loop at a time and multiplying by a factor $\delta=-A^{2}-A^{-2}$. In particular the generator $e_{i}$ is represented thus.

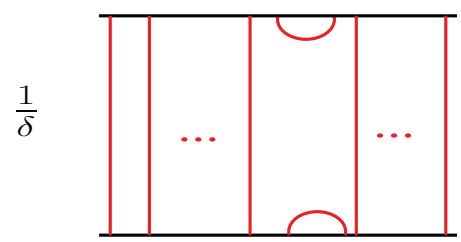

And in the same breath, Kauffman discovered another two-variable knot polynomial ([Kau90]) which is essentially an unoriented link invariant renormalisable just as one goes between the Kauffman bracket and $V_{L}(t)$.

His diagrammatics soon led to the solution of a Tait conjecture about alternating knots; see [Kau87, Mur87, Th87. The Tait conjectures were completely solved more recently by Menasco and Thistlethwaite; see MeTh93.

On the subfactor side, progress was made by Ocneanu and Wenzl. Ocneanu's approach to the HOMFLYPT polynomial saw it as coming from the Hecke algebra ([Jo87]) and isolated a sequence of one-variable polynomials understood to have something to do with $S U(n)$, the polynomial $V_{L}(t)$ being the case $n=2$. Ocneanu and Wenzl (see [HOMFLY85] and [We88] ) used the corresponding Hecke algebras to construct new subfactors of indices $\frac{\sin ^{2} k \pi / \ell}{\sin ^{2} \pi / \ell}$ with the case $S U(2)$ corresponding to the subfactors of index $4 \cos ^{2} \pi / n$ described above. The Temperley-Lieb algebra in this case is seen as a quotient of the Hecke algebra. This in turn suggested that one consider a sequence of one-variable specialisations of the HOMFLYPT polynomial which should be related to $S U(n)$ with $V_{L}(t)$ corresponding to $S U(2)$. The sense in which $S U(n)$ appeared was quite clear in the operator algebra context through the work of Baker and Powers ( $\mathrm{BaPo86}])$ as the $\mathrm{II}_{1}$ factor coming from the fixed points of the infinite tensor product action of $S U(n)$ on the $n \times n$ matrices.

The principal graph of a subfactor emerged, being the graph $A_{n}$ for the subfactors constructed from the Temperley-Lieb algebra. It encodes induction-restriction of bimodules between $N$ and $M$. Ocneanu gave a complete classification of subfactors in index $<4$ by Coxeter graphs of types $A_{n}, D_{2 n}, E_{6}$, and $E_{8}$ and showed that the graphs $D_{2 n+1}$ and $E_{7}$ do not occur as principal graphs. 
To see the principal graph arising, look again at these Bratelli graphs.
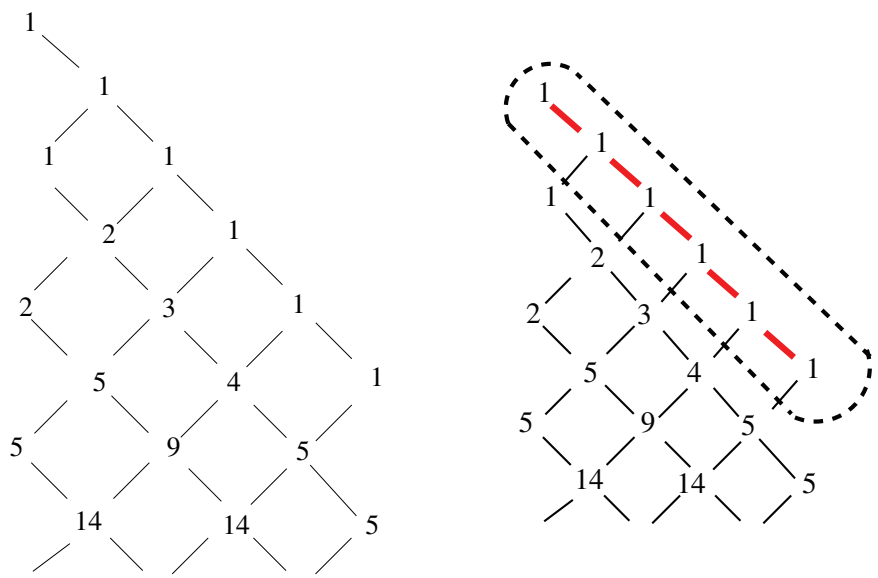

The diagonal chain of 1's is the principal graph for this factor - the Coxeter graph $A_{6}$.

Wassermann considered the full Baker-Powers example for any compact group and privileged unitary representation, and he identified the principal graph from the representation theory - the vertices are all the irreducible representations and the edges occur according to multiplicity of containment of 1 in the tensor product with the privileged representation. The special case of closed subgroups of $S U(2)$ realising the extended Coxeter graphs appeared in GHJ89.

This completes a summary of what happened during the MSRI period 1984-1985 in this area. There was much continuing activity, but the following aspects were unsatisfactory:

i) A "context" was needed for subfactors.

The constructions were all somewhat ad hoc and combinatorial. One would have liked to see the subfactors arising somehow in some other part of operator algebras, especially unitary group representations.

ii) A topological interpretation was needed for $V_{L}(t)$ and the other polynomials.

Conway's skein theory could be adapted to give a genuinely three-dimensional development of all the polynomials but, like the subfactor picture at this time, it was ultimately combinatorial and there was little contact with the rest of lowdimensional topology.

\section{4. $1985-1988$}

For the work we are considering, this year was one of consolidation and organisation. Probably the most notable unifying influence was the theory of quantum groups developed by Jimbo, Woronowicz, and Drinfeld. Quantum groups are noncommutative non-cocommutative Hopf algebras. The tensor product on representations is thus non-commutative, but one requires a commutativity up to isomorphism. It is the intertwiners implementing this weak commutatitivity that contain the rich structural and numerical data - enough to provide lots of new inputs into the Baxter method of solving two-dimensional statistical mechanical models (Ba82 $)$. The three mathematicians above obtained examples of such Hopf algebras as deformations of the enveloping algebras of semisimple Lie algebras. I do not wish to go into the theory any more here, as much has been said and written 
about quantum groups. (For a look at these developments from the point of view of subfactors, see [Jo07.) Suffice it to say that the following picture emerged.

To every irreducible finite-dimensional representation of a simple Lie group/algebra there is

1) A one-parameter family of subfactors. (Sawin Saw95])

2) A knot polynomial with direct statistical mechanical sum formula (Rosso Ro88). For links one can put representations on individual components.

3) A braid group representation with Markov trace.

The values of the deformation parameter " $q$ " of most interest are the $n$th roots of unity, especially those closest to 1 . They are a bit tricky to handle in the quantum group picture as the deformation parameter is no longer a formal variable and semisimplicity is lost. But in the subfactor picture, these roots of unity are singled out by the positivity condition of the trace/inner product, which also takes out all the non-semisimple clutter. (See We98] and [Xu98 for the complete picture.)

The HOMFLYPT two-variable polynomial is related to $s l(n)$ in its identity representation - for each $n$ the corresponding polynomial is a specialisation of HOMFLYPT and put together they determine it. The Kauffman two-variable polynomial is related to the orthogonal and symplectic algebras in the same way.

To jump ahead into the 1990s, it was in the subfactor arena that enduring "sporadic" objects were discovered by Haagerup (and Haagerup and Asaeda, see HaAs99]) of index $\frac{5+\sqrt{13}}{2}$ and principal graphs are illustrated here.

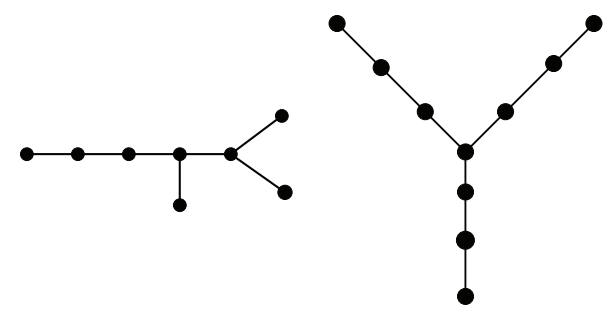

These objects remain "exotic" creatures in the zoo, untouched by quantum groups or any non-von Neumann algebra approach.

During this period 1985-1988, another subject closely related to quantum groups made its presence felt, and that was conformal field theory.

The foundational paper is that of Belavin-Polyakov-Zamolodchikov (see [BPZ80]) and a central example is the treatment of Wess-Zumino-Witten theory in [KZ84. But for those with less physics background, the paper by Tsuchiya and Kanie TK88 was influential. In it they obtained the braid group representations (at roots of unity) that we have discussed, as monodromy for the $n$-point functions in KZ84. This led to the strong suspicion that both subfactors and the knot polynomials were intimately related to conformal field theory.

In an even more murky connection at the time, Fredenhagen, Rehren, and Schroer ([FRS89]) and Longo ([Lo89]), suggested that braid groups and subfactors should crop up in general low-dimensional quantum field theory (QFT), in the Haag-Kastler algebraic QFT framework following Doplicher, Haag, and Roberts (DHR74] and [DHR71]). In this approach to QFT one supposes that to suitable regions of space-time there are von Neumann algebras of observables "localised" in the regions. These algebras are subject to certain general axioms, perhaps the 
most important of which is that observables localised in regions of space-time which cannot be connected by a message going at less than the speed of light have to commute. It is remarkable how much structure can be obtained from this axiomatic approach. See for instance the book by Haag [Ha96].

The idea of Doplicher, Haag, and Roberts was to investigate "statistics" of quantum fields by examining how they behave when their support regions are interchanged. This led to representations of the symmetric group and proper endomorphisms of von Neumann algebras. Fredenhagen, Rehren, Schroer, and Longo proposed that in lower dimensions the braid group would replace the symmetric group and the endomorphisms should be ones onto the subfactors we have discussed above.

\section{1988, Atiyah, Segal, Witten}

Segal had proposed a penetrating system of axioms for conformal field theory based on a cobordism idea, and Atiyah and Witten developed a version which should work in a purely topological situation where the Segal theory required a complex structure. The two theories, conformal and topological, were closely related in that the gross structure of a conformal field theory provides a topological quantum field theory in dimension three. I do not want to go into all this here as a lot has been said and written about TQFT. But at least it has to be said that an extraordinary formal expression of this theory was obtained by Witten [Wt89] giving an interpretation of $V_{L}(t)$, at $t=e^{2 \pi i / k}$, as a functional integral in a $(2+1)$-dimensional gauge theory, gauge group $S U(2)$ (as expected), with Chern-Simons action, the knot invariant being the expected value of the trace of the monodromy along the components of the link which now became known as "Wilson loops".

Here is Witten's formula in all its glory:

$$
V_{L}\left(e^{2 \pi i /(\ell+2)}\right)=\int D \mathfrak{A} \exp \left(\frac{i \ell}{4 \pi} \int_{S^{3}} \operatorname{Tr}\left(A \wedge d A+\frac{2}{3} A \wedge A \wedge A\right)\right) W,
$$

where the integral is over all Lie algebra valued 1-forms modulo gauge transformations and $W$ is the product of the traces of parallel transport using $A$ along each of the components of the link, each one being assigned the two-dimensional representation of $S U(2)$.

The functional integral in the above formula may or may not exist but it is the role of TQFT to provide a context for such a beast. Replacing $S^{3}$ in Witten's formula by any 3-manifold gives an invariant of a link in that manifold - why not? To the unbeliever, the formula itself could be taken as mumbo-jumbo, but the TQFT reading of it provided real pay dirt in the form of an explicit formula for the extension of $V_{L}(t)$ to links in an arbitrary 3-manifold via surgery on links or Heegard splitting! These formulae were completely verified by Reshetikhin and Turaev in RT91 so that the unbeliever had no way to doubt that Witten's formula had meaning. The more so because Witten attacked the formula by the muchused perturbative methods of quantum field theory to obtain another approach intimately linked to the Vassiliev theory we shall mention briefly below. Also, building on work of Lickorish ([Li97]), Blanchet, Habegger, Masbaum, and Vogel in BHMV92 developed all the ingredients of a TQFT (vector spaces for surfaces, vectors in those spaces when the surface bounds a 3 -manifold ...) in a hands-on way simply by considering systems of links in 3-manifolds with boundary subject 
to the Kauffman bracket relation and a root of unity condition (which, remember, came straight from the subfactor analysis of the Temperley-Lieb algebra).

A slightly simpler version of the TQFT framework was applied by Turaev and Viro TV92] and Ocneanu Oc87] to obtain 3-manifold invariants from general categorical data as provided, for instance, by a subfactor - where the required positiviity was built in. Thus, to every subfactor with a finite principal graph (such as those of Haagerup and Haagerup-Asaeda) there is a TQFT. The meaning of these theories remains somewhat obscure in general, though they can give classical invariants like enumerating representations of the fundamental group of the manifold. Taking the abstraction one level further, Calegari, Freedman, and Walker in CFW08 have shown that TQFTs are powerful enough to separate 3-manifolds, though in dimension 4 there are counterexamples to such an approach.

\section{MSRI MEETING JANUARY 1989 AND THE WORK OF WASSERMANN}

In January 1989 there was a workshop at MSRI which brought together many of the key actors in the above stories, for instance Atiyah, Bott, Witten, Jimbo, Miwa, Kauffman, Faddeev, Wassermann.... This meeting greatly clarified the situation, and those of us who had felt out of the loop finally got over the feeling that the Russians and the Japanese knew something that we didn't. As a direct result of this meeting, the subfactor side of the picture finally opened up. Wassermann and I were struggling to interpret the $e_{i}$ subfactors as fixed-point algebras in the spirit of Baker-Powers but in the context of conformal field theory where loop groups replaced compact groups as clearly suggested by Pressley and Segal in PS86. After many false leads we came up with a conjecture in the theory of loop group representations only to realise that it was entirely in the spirit of Fredenhagen, Rehren, and Schroer with endomorphisms, braid groups and all! The conjecture was proved completely by Wassermann in the more general $S U(n)$ case; see Wa98. Here is the result for $S U(2)$. I give some detail as not enough has been said or written about this beautiful theory.

The loop group $L S U(2)$ is the group of all $C^{\infty}$ maps (loops) from the circle $S^{1}$ to the compact simply connected group $S U(2)$. (A first shot at the Lie algebra of this group would be functions to the Lie algebra of $S U(2)$ which are polynomials in the circle variable $z$.) $L S U(2)$ has a beautiful family of irreducible infinite-dimensional unitary projective representations which are characterised by the fact that they extend to the obvious semidirect product of $L S U(2)$ and $S^{1}$ in such a way that the Fourier decomposition of the representation has only positive Fourier modes ("positivity of the energy"). This "discrete series" of representations is parametrised by two discrete variables: (i) the "level" $\ell$ which determines the central extension of $L S U(2)$ implicated in the projective representation; and (ii) the "spin" $j$, the usual half-integer used to parametrise the irreducible representations of $S U(2)$, which occur as the fixed points for the action of $S^{1}$ on the Hilbert space.

(On the Lie algebra level the extensions to the semidirect product with $S^{1}$, and the central extension required to handle projective representations, define precisely the affine Kac-Moody Lie algebra corresponding to $s l(2)$.)

It is of considerable importance that for a given $\ell$, only finitely many values of $j$ arise, in fact $2 j \leq l$. This means that for fixed $\ell$ the spins could be thought of as the vertices of a Coxeter graph $A_{\ell+2}$, hinting at a connection with the principal graph of a subfactor. 
If one takes a closed interval $I$ on the circle, let $I^{c}$ denote the closed interval which is the closure of the complement of $I$ and let $L_{I}$ be the subgroup of loops which are the identity on $I^{c}$. If $\pi$ on $\mathfrak{H}_{j}$ is one of the discrete series representations above, $\pi\left(L_{I}\right)$ and $\pi\left(L_{I}\right)$ commute with each other. (Because $\pi$ is projective this is not obvious - this is where simple connectedness comes in.) If one can show that the von Neumann algbera $L_{I}^{\prime \prime}$ generated by $\pi\left(L_{I}\right)$ is a factor, then we have a subfactor

$$
\pi\left(L_{I}\right)^{\prime \prime} \subseteq \pi\left(L_{I^{c}}\right)^{\prime}
$$

(as always in von Neumann algberas ' denotes the commutant of a set of operators).

Wassermann showed that as $\ell$ and $j$ vary, these subfactors realise the values $\frac{\sin ^{2} y \pi /(\ell+2)}{\sin ^{2} \pi /(\ell+2)}$, and much more; see Wa98.

To be able to get started, it is crucial that the representations involved be manifestly unitary. For this the well-developed theory of representations of $\mathrm{C}^{*}$-algebras is available. One begins with the simple-minded unitary repsentation of $L S U(2)$ on $L\left(S^{1}\right) \otimes V_{j}, V_{j}$ being the spin $j$ representation of $S U(2)$. One then applies fermionic second quantisation- $L S U(2)$ acts functorially on the $\mathrm{C}^{*}$-algebra of the canonical anticommutation relations for this Hilbert space. This algebra has an irreducible representation constructed from the "quasi-free" state with covariance operator being the projection $P$ onto holomorphic functions. A loop group element preserves the equivalence class of this representation of the CAR algebra iff its commutator with $P$ is Hilbert-Schmidt. Thus we get a unitary projective representation of all loops with this commutator property which turns out to be a "half-differentiability" property. All the discrete series representations can be found in these ones and their tensor powers.

The most difficult technical part of the proof is simply to prove finite index of the subfactor. Wassermann achieves this by using the Connes tensor product of Hilbert spaces over von Neumann algebras ( $\mathrm{Co94}$ ) to construct what is known as the "fusion" of two discrete series representations of $L S U(2)$ to produce another of the same level. One shows that all representations with fixed level are unitarily equivalent upon restriction to an $L_{I}$. By identifying $I$ of one representation with $I^{c}$ of another, one may form the Connes tensor product $\mathfrak{H}_{j_{1}} \otimes_{\left\{L_{I}\right\}^{\prime \prime}} \mathfrak{H}_{j_{2}}$ which inherits a bimodule structure over $\left\{L_{I}\right\}^{\prime \prime}-\left\{L_{I^{c}}\right\}^{\prime \prime}$. One shows that this bimodule structure is in fact the restriction of and action of all of $L S U(2)$ to obtain the fused representation.

To do this extension to $L S U(2)$, Wassermann observed that the definition of the Connes tensor product actually uses the four-point functions of conformal field theory and identifies the $\left\{L_{I}\right\}^{\prime \prime}-\left\{L_{I^{c}}\right\}^{\prime \prime}$ bimodule using the Kniznik-Zamolodchikov equation!

The connection with quantum field theory is as follows. The circle $S_{1}$ is a onedimensional space-time and the algebras $\pi\left(L_{I}\right)^{\prime \prime}$ are the algebras of observables localised in the interval $I$. Two intervals are causally separated if they are disjoint. So in fact this is an instance of the Fredenhagen-Rehren-Schroer theory, with braiding and endomorphisms as predicted!

\section{Some more RECEnt DeVElopments}

We record some bullet points among many developments in these areas beyond what we have discussed above. 
- Turning again to topology, a new ingredient entered the game in the early 1990's - Vassiliev theory. Thinking of knots as smooth functions from $S^{1}$ to $\mathbb{R}^{3}$, in Va95, Vassiliev began the study of the algebraic topology of this space and obtained new knot invariants. Birman and Lin furthered this study and the concept of a "finite type invariant" emerged; see BL93. The idea is to extend any additive knot invariant Inv to an invariant of immersed curves with at worst double point singularities by locally setting

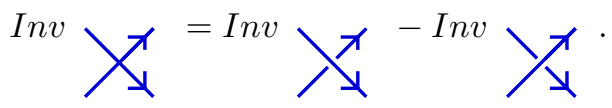

Then Inv is of finite type $n$ if it vanishes on immersed curves with $n$ double points.

The knot polynomials are a source of finite-type invariants but it was shown by Vogel in Vo01 that there are others. Kontsevich discovered a beautiful general integral formula for finite-type invariants, thinking of them more as a perturbative expansion of a functional integral; see [Kon93].

Bar-Natan put everything together in a stunningly comprehensible paper BN95] which made the whole theory broadly accessible and showed that one could construct knot invariants by studying "chord diagrams" modulo certain linear relations.

All this has to a certain extent been extended to knots and links in arbitrary 3 -manifolds, though I believe that, beyond homology 3 -spheres, things remain a little murky.

- Another major problem in this area is to understand how the Witten-Reshetikhin-Turaev invariant depends on the root of unity. In the best of all worlds, there would be a holomorphic function of $q$ of which it is the boundary value, but this appears to be too naive. Recent progress has been made by Garoufalidis Ga07.

There are various conjectures concerning the asymptotic behaviour of the WittenReshetikhin-Turaev invariants and how they relate to other geometric or topological invariants. Let me mention Kashaev's volume conjecture (just for knots); see Kash96. If $K$ is a knot with hyperbolic complement, one looks at

$$
V_{S_{2}}, k \text { dimensional representation }\left(e^{\pi i / k}\right)
$$

(the value of $t$ is the value for which the invariant of an unknot is zero (!)). Then as $k \rightarrow \infty$, the asymptotic growth of this number is controlled by the hyperbolic volume. The result is known for some small knots by explicit computations. The experts are not entirely in agreement as to how it should be true.

- The question of linearity of the braid group was settled by Bigelow and Krammer ( $\mathrm{Bi01}$, Kr02]) by showing that the braid group representation associated with the two-variable Kauffman polynomial is faithful. It remains open whether the representation in the Temperley-Lieb algebra is faithful (for generic values of the parameter).

- A more recent development has been Khovanov homology ([Khov00]). This uses the basic Kauffman resolution $\backslash \longrightarrow \sim_{+}^{+}$) of a crossing but "categorifies" it in that the basic invariant associated to a link is a graded complex and the relation between the complexes at a crossing is an exact sequence between the three complexes. The invariant ends up, at least in the hands of Bar-Natan, being a complex up to homotopy equivalence. The Euler characteristic of the complex is $V_{L}(t)$. Bar-Natan's version is highly calculable by a "planar algebra" approach. 
Khovanov and others have extended categorification to many other knot invariants and beyond. It is strictly more powerful than the corresponding Euler characteristic polynomials, and it looks like it is the arena for connections with gauge theory, e.g. Floer homology; see Ras05].

What about subfactors?

The 1990's saw deep classification results by Sorin Popa who showed that in "amenable" cases (both the factor, the subfactor, and the principal graph), the subfactor is completely classified by what is known as the "standard invariant" (an enrichment of the principal graphs containing not just the bimodules and their tensor powers but intertwiners between them). These results extend Connes' breakthrough results from the 1970's classifying automorphisms and group actions ( Po94, Co75]).

The subfactors of index $<4$ have the Coxeter graphs A-D-E as principal graphs and can be thought of as some kind of quantum truncation of the finite subgroups of $S U(2)$. Zuber et al. found some analogues of such behaviour for finite subgroups of $S U(3)$; see DFZ90. Ocneanu gave complete results for $S U(3)$ and higher in Oc00. This gives a rich family of subfactors with finite principal graphs, falling into several series and exceptional cases.

Bisch and I investigated the implications of the existence of an intermediate subfactor and discovered a Temperley-Lieb generalisation called the Fuss-Catalan algebra; see [BiJo97. It was used to give statistical mechanical models in diF98. but seems to have nothing to do with knot theory. Severe restrictions on the positions of more than just one intermediate subfactor were discovered in GrJ07.

I spent many years with some but little success in an attempt to use subfactors to provide information on a large variety of combinatorial objects. Things like Latin squares and Hadamard matrices can be used to construct subfactors and the hope still is that the subfactor invariants - the standard invariant referred to above - can be exploited to say something about the combinatorial object (which may or may not possess ordinary group symmetry). The stumbling block is computational-for instance, how do you compute the principal graph? If one only wants information near the beginning of the principal graph there is no problem, but the computational complexity of the calculation seems to grow exponentially the further out one goes. Even the first couple of relative commutants did give some interesting information; see BBJ97. This program may eventually prove fruitful, but one thing it did lead to was the development of planar algebras which provided a very convenient axiomatisation of the standard invariant of a subfactor and a useful technique for analysing their structure. It is similar in spirit to Conway's "skein theory".

- Planar algebra is based on the notion of a planar tangle. Here is an example:

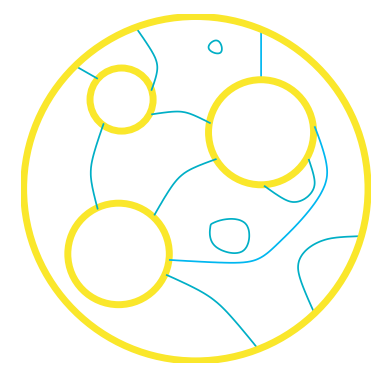


The idea is that a planar algebra is a (graded) vector space $P_{n}$ whose elements may be used as "inputs" of any planar tangle, which responds with an output. It is a theorem (Jon99]) that the standard invariant of a subfactor is a planar algebra. So given $A, B, C$ in the standard invariant (i.e. each one in $P_{n}$ for some $n$ which may differ for $A, B$ and $C$ ) in this case in $P_{2}, P_{3}$, and $P_{2}$, respectively, the picture

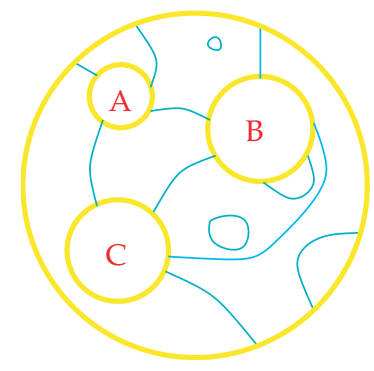

represents another element of the standard invariant (in this case in $P_{3}$ ). As another example the tangle below gives an associative algebra structure $A B$ on each $P_{n}\left(P_{4}\right.$ in the example):

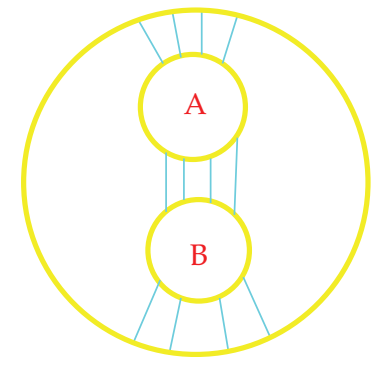

Given a planar algebra there is a well-known way to (try to) construct a factor and subfactor whose standard invariant is the given planar algebra. One uses the above algebra structure on each $P_{n}$, embeds them unitally one in the next, and takes a completion of the union. It works well in the "finite depth" case (where the dimension-generating function for the $P_{n}$ 's is rational), but not in general. Popa in Po95, gave another construction which works in general but produces a rather different kind of $\mathrm{II}_{1}$ factor. In a recent interaction with random matrices and Voiculescu's free probability, an alternative to Popa's construction was found involving a graded product on the planar algebra; see GJS07.

- Finally, we mention the approach of Freedman et al. to building a quantum computer ([FKLW03]). The main technical difficulty with the usual qubit approach is to get a large enough system of quantum bits - be they electrons, protons, ions, or whatever - to interact strongly with each other and not the environment for the duration of the calculation. The idea is to overcome this problem (and some error-correcting difficulties) by spreading the calculation out in a two-dimensional quantum fluid and replacing qubits by quasi-particles. Since the system is twodimensional, interchanging the quasi-particles will be governed by a unitary braid group representation, most likely at first one of the ones we have discussed as they are the simplest. By observing the evolution of an appropriate braid, one may do a computation, and in FKLW03] it is shown that, if the braid group representation is 
the one we have described for an index $4 \cos ^{2} \pi / 5$ subfactor, any computation that could be done by any other quantum computer could be done using such a quantum fluid. It is a fascinating question simply in physics to know if such "non-abelian anyonic" quantum systems exist. If they do, they will surely be observed first at extremely low temperatures.

In spite of all the progress in and around TQFT one basic question remains as open today as it was in 1984: is there a knot $K$ with $V_{K}(t)=1$ ?

Note though that the problem for links with more than one component was solved by Thistlethwaite in Th01]; see also [EKT03.

\section{About the AUthor}

Vaughan Jones is a professor at the University of California at Berkeley. Among his many honors and awards are the Fields Medal (1990), Fellow of the Royal Society, and Member of the National Academy of Sciences.

\section{REFERENCES}

[HaAs99] M. Asaeda, U. Haagerup, Exotic subfactors of finite depth with Jones indices $(5+\sqrt{13}) / 2$ and $(5+\sqrt{17}) / 2$, Comm. Math. Phys. 202 (1999), 1-63. MR.1686551 (2000c:46120)

[BaPo86] B. M. Baker, R. T. Powers, Product states of certain group-invariant AF-algebras, J. Operator Theory 16 (1986), no. 1, 3-50. MR847331 (87m:46117)

[BN95] Dror Bar-Natan, On the Vassiliev knot invariants, Topology 34 (1995), no. 2, 423-472. MR1318886 (97d:57004)

[Bi75] J. Birman, Braids, links and mapping class groups, Princeton University Press, 1975. MR0375281 (51:11477)

[BL93] Joan S. Birman, Xiao-Song Lin, Knot polynomials and Vassiliev's invariants, Invent. Math. 111 (1993), no. 2, 225-270. MR1198809 (94d:57010)

[Ba82] R. Baxter, Exactly solved models in statistical mechanics, Academic Press (Harcourt Brace Jovanovich, Publishers), London, 1982. MR690578(86i:82002a)

[BPZ80] A. A. Belavin, A. M. Polyakov, and A. B. Zamolodchikov, Infinite conformal symmetry in two-dimensional quantum field theory, Nuclear Physics B 241 (1980), 333-380. MR757857 (86m:81097)

[BBJ97] Eiichi Bannai, Etsuko Bannai, François Jaeger, On spin models, modular invariance, and duality, J. Algebraic Combin. 6 (1997), 203-228. MR1456578(98f:05150)

[Bi01] Stephen J. Bigelow, Braid groups are linear, J. Amer. Math. Soc. 14 (2001), 471486. MR1815219 (2002a:20043)

[BiJo97] D. Bisch and V. F. R. Jones, Algebras associated to intermediate subfactors, Inventiones Mathematicae 128 (1997), 89-157. MR1437496 (99c:46072)

[BHMV92] C. Blanchet, N. Habegger, G. Masbaum, P. Vogel, Three-manifold invariants derived from the Kauffman bracket, Topology 31 (1992), no. 4, 685-699. MR1191373 (94a:57010)

[CFW08] Danny Calegari, Michael Freedman, and Kevin Walker, Positivity of the universal pairing in 3 dimensions, arXiv:0802.3208

[Co94] A. Connes, Noncommutative geometry, Academic Press, San Diego, 1994. MR.1303779 (95j:46063)

[Co75] A. Connes, Outer conjugacy classes of automorphisms of factors, Annales Scientifiques de l'École Normale Supérieure 8 (1975), 383-419. MR0394228 (52:15031)

[DHR74] S. Doplicher, R. Haag, and J. E. Roberts, Local observables and particle statistics. II. Comm. Math. Phys. 35 (1974), 49-85. MR0334742 (48:13060)

[DHR71] S. Doplicher, R. Haag, and J. E. Roberts, Local observables and particle statistics. I. Comm. Math. Phys. 23 (1971), 199-230. MR0297259 (45:6316) 
[EKT03] Shalom Eliahou, Louis H. Kauffman, and Morwen B. Thistlethwaite, Infinite families of links with trivial Jones polynomial, Topology 42 (2003), no. 1, 155-169. MR.1928648 (2003g:57015)

[FW87] J. Franks and R. F. Williams, Braids and the Jones polynomial, Trans. Amer. Math. Soc. 303 (1987), 97-108. MR896009 (88k:57006)

[DFZ90] P. Di-Francesco and J.-B. Zuber, "SU(N) lattice integrable models and modular invariance", Recent developments in conformal field theories, 179-216, World Scientific, 1990. MR 1160374

[diF98] P. di Francesco, New integrable lattice models from Fuss-Catalan algebras, Nuclear Phys. B 532 (1998), no. 3, 609-634. MR1657030 (99k:82020)

[FRS89] K. Fredenhagen, K.-H. Rehren, and B. Schroer, Superselection sectors with braid group statistics and exchange algebras, Communications in Mathematical Physics 125 (1989), 201-226. MR1016869 (91c:81047)

[FKLW03] M. Freedman, A. Kitaev, M. Larsen, and Wang Zhenghan, Topological quantum computation. Mathematical challenges of the 21st century (Los Angeles, CA, 2000), Bull. Amer. Math. Soc. (N.S.) 40 (2003), no. 1, 31-38. MR.1943131 (2003m:57065)

[Ga07] Stavros Garoufalidis, Chern-Simons theory, analytic continuation and arithmetic, arXiv:0711.1716

[GrJ07] Pinhas Grossman and Vaughan F. R. Jones, Intermediate subfactors with no extra structure, J. Amer. Math. Soc. 20 (2007), no. 1, 219-265. MR2257402 (2007h:46077)

[GJS07] A. Guionnet, V. F. R. Jones, and D. Shlyakhtenko, Random matrices, free probability, planar algebras and subfactors, arXiv:0712.2904

[Ha96] R. Haag, Local quantum physics, Springer-Verlag, Berlin-Heidelberg-New York, 1996. MR1405610 (98b:81001)

[HOMFLY85] P. Freyd, D. Yetter, J. Hoste, W. Lickorish, K. Millet, and A. Ocneanu, A new polynomial invariant of knots and links, Bull. Amer. Math. Soc. 12 (1985), 239246. MR776477 (86e:57007)

[GHJ89] F. Goodman, P. de la Harpe, and V. F. R. Jones, Coxeter graphs and towers of algebras, MSRI Publications (Springer), vol. 14, 1989. MR999799 (91c:46082)

[GJS07] A. Guionnet, V. F. R. Jones, and D. Shlyakhtenko, Random matrices, free probability, planar algebras and subfactors, arXiv:0712.2904

[Jon83] V. F. R. Jones, Index for subfactors, Invent. Math. 72 (1983), 1-25. MR696688 (84d:46097)

[Jon99] V. F. R. Jones, Planar algebras, Preprint, Berkeley, 1999, math.QA/9909027.

[Jo85] V. F. R. Jones, A polynomial invariant for knots via von Neumann algebras, Bull. Amer. Math. Soc. 12 (1985), no. 1, 103-111. MR766964 (86e:57006)

[Jo87] V. F. R. Jones, Hecke algebra representations of braid groups and link polynomials, Annals of Mathematics 126 (1987), 335-388. MR.908150 (89c:46092)

[Jo07] Vaughan F. R. Jones, "In and around the origin of quantum groups", Prospects in mathematical physics, 101-126, Contemp. Math., 437, Amer. Math. Soc., Providence, RI, 2007. MR2354658 (2008k:17018)

[Kash96] Rinat M. Kashaev, "Quantum hyperbolic invariants of knots", Discrete integrable geometry and physics (Vienna, 1996), 343-359, Oxford Lecture Ser. Math. Appl., 16, Oxford Univ. Press, New York, 1999. MR1676604 (2001a:57021)

[Kau87] L. H. Kauffman, State models and the Jones polynomial, Topology 26 (1987), 395407. MR899057 (88f:57006)

[Kau90] L. H. Kauffman, An invariant of regular isotopy, Trans. Amer. Math. Soc. 318 (1990), no. 2, 417-471. MR958895 (90g:57007)

[Khov00] Mikhail Khovanov, A categorification of the Jones polynomial, Duke Math. J. 101 (2000), no. 3, 359-426. MR 1740682 (2002j:57025)

[KZ84] V. Knizhnik and A. Zamolodchikov, Current algebra and Weiss-Zumino models in two dimensions, Nuclear Physics B 247 (1984), 83-103. MR853258 (87h:81129)

[Kon93] Maxim Kontsevich, "Vassiliev's knot invariants", I. M. Gelfand Seminar, 133150, Adv. Soviet Math., 16, Part 2, Amer. Math. Soc., Providence, RI, 1993. MR1237836 (94k:57014)

[Kr02] Daan Krammer, Braid groups are linear, Ann. of Math. 155 (2002), 131-156. MR1888796 (2003c:20040) 
[LM86] W. B. R. Lickorish and K. C. Millett, The reversing result for the Jones polynomial, Pacific J. Math. 124 (1986), no, 1, 173-176. MR850674 (87k:57007)

[Li97] W. B. R. Lickorish, An introduction to knot theory, Graduate Texts in Mathematics, 175, Springer-Verlag, New York, 1997. MR1472978 (98f:57015)

[Lo89] R. Longo, Index of subfactors and statistics of quantum fields, I., Communications in Mathematical Physics 126 (1989), 217-247. MR1027496 (91c:46097)

[MeTh93] W. Menasco and M. Thistlethwaite, The classification of alternating links, Ann. Math. 138 (1993), 113-171. MR.1230928 (95g:57015)

[Mor86] H. R. Morton, Seifert circles and knot polynomials, Math. Proc. Cambridge Philos. Soc. 99 (1986), 107-109. MR809504 (87c:57006)

[Mur87] K. Murasugi, The Jones polynomial and classical conjectures in knot theory, Topology 26 (1987), 187-194. MR895570 (88m:57010)

[Oc87] A. Ocneanu, "Quantized group, string algebras and Galois theory for algebras", Operator algebras and applications, vol. 2, 119-172, L.M.S Lecture Note Series, 136, 1987. MR 996454 (91k:46068)

[Oc00] A. Ocneanu, "The classification of subgroups of quantum $\mathrm{SU}(N)$ ", Quantum symmetries in theoretical physics and mathematics (Bariloche, 2000), 133-159, Contemp. Math., 294, Amer. Math. Soc., Providence, RI, 2002. MR 1907188 (2003h:81101)

[Po94] S. Popa, Classification of amenable subfactors of type II, Acta Mathematica 172 (1994), 163-255. MR:1278111 (95f:46105)

[Po95] S. Popa, An axiomatization of the lattice of higher relative commutants of a subfactor, Inventiones Mathematicae 120 (1995), 427-446. MR1334479 (96g:46051)

[Ras05] Jacob Rasmussen, "Knot polynomials and knot homologies", Geometry and topology of manifolds, 261-280, Fields Inst. Commun., 47, Amer. Math. Soc., Providence, RI, 2005. MR2189938(2006i:57029)

[RT91] N. Yu. Reshetikhin and V. G. Turaev, Invariants of 3-manifolds via link polynomials and quantum groups, Inventiones Mathematicae 103 (1991), 547-598. MR1091619 (92b:57024)

[Ro88] M. Rosso, Groupes quantiques et modèles à vertex de V. Jones en théorie des nœuds. (French) [Quantum groups and V. Jones's vertex models for knots], C. R. Acad. Sci. Paris Ser. I Math. 307 (1988), no. 6, 207-210. MR956807 (90d:57009)

[Saw95] S. Sawin, Subfactors constructed from quantum groups, Amer. J. Math. 117 (1995), 1349-1369. MR1363071 (96h:46097)

[PS86] A. Pressley and G. Segal, Loop groups, Oxford University Press, 1986. MR900587 (88i:22049)

[TL70] H. N. V. Temperley and E. H. Lieb, Relations between the "percolation" and "colouring" problem and other graph-theoretical problems associated with regular planar lattices: some exact results for the "percolation" problem, Proc. Roy. Soc. London Ser. A 322 (1971), no. 1549, 251-280. MR0498284 (58:16425)

[Th87] M. Thistlethwaite, A spanning tree expansion of the Jones polynomial, Topology 26 (1987), 297-309. MR899051 (88h:57007)

[Th01] Morwen Thistlethwaite, Links with trivial Jones polynomial, J. Knot Theory Ramifications 10 (2001), no. 4, 641-643. MR1831681 (2002a:57012)

[TK88] A. Tsuchiya and Y. Kanie, Vertex operators on conformal field theory on $\mathbf{P}^{1}$ and monodromy representations of braid groups, Advanced Studies in Pure Mathematics 16 (1988), 297-372. MR972998(89m:81166)

[TV92] V. G. Turaev and and O. Y. Viro, State sum invariants of 3-manifolds and quantum 6j-symbols, Topology 31 (1992), 865-902. MR.1191386 (94d:57044)

[Va95] Victor A. Vassiliev, "Topology of discriminants and their complements", Proceedings of the International Congress of Mathematicians, Vol. 1, 2 (Zürich, 1994), 209-226, Birkhäuser, Basel, 1995. MR1403923 (97j:57041)

[Vo01] Pierre Vogel, "Invariants de type fini", Nouveaux invariants en gèomètrie et en topologie, 99-128, Panor. Synthéses, 11, Soc. Math. France, Paris, 2001. MR.1882446 (2002j:57028)

[Wa98] A. Wassermann, Operator algebras and conformal field theory. III. Fusion of positive energy representations of $\mathrm{LSU}(N)$ using bounded operators, Invent. Math. 133 (1998), no. 3, 467-538. MR1645078 (99j:81101) 
[We98] H. Wenzl, $C^{*}$ tensor categories from quantum groups, J. Amer. Math. Soc. 11 (1998), 261-282. MR1470857 (98k:46123)

[We88] H. Wenzl, Hecke algebras of type $A_{n}$ and subfactors, Invent. Math. 92 (1988), 349-383. MR936086 (90b:46118)

[We87] H. Wenzl, On sequences of projections, C. R. Math. Rep. Acad. Sci. Canada, 9 (1987), no. 1, 5-9. MR873400 (88k:46070)

[Wt89] E. Witten, Quantum field theory and Jones polynomial. Comm. Math. Phys. 121 (1989), 351-399. MR0990772 (90h:57009)

[Wo87] S. L. Woronowicz, Twisted SU(2) group. An example of a noncommutative differential calculus, Publ. Res. Inst. Math. Sci. 23 (1987), no. 1, 117-181. MR890482 (88h:46130)

[Xu98] Xu F., Standard $\lambda$-lattices from quantum groups, Inventiones Mathematicae 134 (1998), 455-487. MR.1660937 (2000c:46123)

Department of Mathematics, University of California at Berkeley, Berkeley, CalIFORNIA 94720

E-mail address: vfr@math.berkeley.edu 